\title{
Berberine induces mitochondrial-mediated apoptosis and protective autophagy in human malignant pleural mesothelioma NCI-H2452 cells
}

\author{
ZHOUHONG YAO ${ }^{1}$, YUNYAN WAN ${ }^{1}$, BIN LI $^{2}$, CONGCONG ZHAI $^{1}$, FEI YAO ${ }^{3}$, \\ YANMENG KANG ${ }^{4}$, QINGHUA LIU ${ }^{5}$ and DIANJIE LIN ${ }^{1}$ \\ Departments of ${ }^{1}$ Respiratory Medicine, ${ }^{2}$ Health Care of Respiratory Medicine and ${ }^{3}$ Thoracic Surgery, \\ Shandong Provincial Hospital Affiliated to Shandong University; ${ }^{4}$ Department of Respiratory Medicine, \\ Shandong Provincial Qianfoshan Hospital, Jinan, Shandong 250021; \\ ${ }^{5}$ Department of Respiratory Medicine, Shanghai East Hospital, Tongji University, \\ Shanghai 200120, P.R. China
}

Received March 11, 2018; Accepted September 19, 2018

DOI: $10.3892 /$ or.2018.6757

\begin{abstract}
Increasing evidence shows that berberine has antitumor effects against a number of tumor cells. In the present study, we evaluated the effect of berberine on the proliferation of the human malignant pleural mesothelioma (MPM) cell line NCI-H2452, and explored the therapeutic potential and underlying mechanisms of this agent. Our results showed that berberine inhibited the proliferation of NCI-H2452 cells in a dose- and time-dependent manner and could induce apoptosis, possibly through a caspase-9-dependent intrinsic mitochondrial pathway. In addition, autophagy was induced by berberine, which was characterized by the accumulation of LC3-II and decreased p62 expression. We used inhibitors of apoptosis and autophagy, and an inducer of autophagy, to evaluate the significance of autophagy in berberine-induced cell death. The results demonstrated that apoptosis is the primary route through which berberine induces NCI-H2452 cell death. Berberine-induced autophagy may be an adaptive response to antitumor agents and have a protective role in MPM cells. Inhibition of autophagy enhanced berberine-induced apoptosis. Therefore, inhibition of autophagy may be an effective treatment strategy in the management of MPM. In conclusion, berberine is a potent antitumor agent for treating
\end{abstract}

Correspondence to: Dr Qinghua Liu, Department of Respiratory Medicine, Shanghai East Hospital, Tongji University, 150 Jimo Road, Pudong New Area, Shanghai 200120, P.R. China

E-mail: 1zlqhlz@163.com

Dr Dianjie Lin, Department of Respiratory Medicine, Shandong Provincial Hospital Affiliated to Shandong University, 324 Jingwuweiqi Road, Jinan, Shandong 250021, P.R. China

E-mail: dianjielin@126.com

Key words: berberine, apoptosis, malignant pleural mesothelioma, autophagy
MPM, and it induces mitochondrial-mediated apoptosis and protective autophagy in human NCI-H2452 MPM cells.

\section{Introduction}

Malignant pleural mesothelioma (MPM) is a highly aggressive malignancy associated with environmental exposure to asbestos (1). The worldwide incidence of this fatal disease is expected to increase in the near future (2). Despite recent advances in clinical treatments, including surgery, chemotherapy, radiotherapy and trimodality therapy, these methods do not have good curative effects and have many undesirable side effects. MPM remains difficult to treat and standard therapies are still in flux. Therefore, novel and more effective treatment strategies are urgently required.

Berberine is an isoquinoline alkaloid exacted from a number of medicinal plant species such as Berberis aquifolium and Berberis aristata, with active antibacterial effects. Berberine has extensive pharmacological effects, including anti-hypertensive, anti-diabetic, anti-arrhythmia and anti-hyperlipidemic (3-6). Recent studies have shown that berberine exerts antitumor effects in a variety of human cancer cells, such as breast cancer, hepatocellular carcinoma, leukemia, and esophageal cancer, both in vitro and in vivo, by suppressing proliferation, inducing apoptosis, arresting the cell cycle, and inhibiting invasion and metastasis (7-12). In addition, it has been recently reported that berberine-induced autophagy has been observed in hepatic and colon cancer cells $(13,14)$. These findings indicated that berberine is a promising agent for clinical application in cancer treatment.

However, to the best of our knowledge, the antitumor effects of berberine have not been investigated in MPM cells. Therefore, we aimed to investigate the antitumor effects of berberine in human MPM NCI-H2452 cells, and to characterize the roles of berberine-induced apoptosis and autophagy, and the underlying molecular mechanisms, in the antitumor activity of the drug. 


\section{Materials and methods}

Reagents. Berberine was obtained commercially from Sigma-Aldrich (Merck KGaA, Darmstadt, Germany). It was dissolved in dimethyl sulfoxide (DMSO; Beijing Solarbio Science \& Technology Co., Ltd., Beijing, China), and then diluted in HyClone ${ }^{\mathrm{TM}}$ RPMI-1640 medium (GE Healthcare Life Sciences, Logan, UT, USA) to the desired concentrations. All concentrations contained a final DMSO concentration of $<0.1 \%$, not enough to affect cell growth. MTT was also purchased from Sigma-Aldrich (Merck KGaA). The PE Annexin V Apoptosis Detection kit was purchased from BD Biosciences (San Diego, CA, USA). Rapamycin, z-VAD-fmk and 3-methyladenine (3-MA) were purchased from Cayman Chemical Company (Ann Arbor, MI, USA).

Cell culture. The human MPM NCI-H2452 cell line was purchased from the Shanghai Institute of Cell Biology, the Chinese Academy of Sciences (Shanghai, China) and cultured in RPMI-1640 medium (HyClone Laboratories; GE Healthcare Life Sciences) supplemented with $10 \%$ fetal bovine serum (FBS) (Shanghai ExCell Biology, Inc., Shanghai, China), $100 \mathrm{mg} / \mathrm{ml}$ streptomycin and $100 \mathrm{U} / \mathrm{ml}$ penicillin at $37^{\circ} \mathrm{C}$ in a humidified atmosphere containing $5 \% \mathrm{CO}_{2}$.

Cell viability assay. The effect of berberine on NCI-H2452 cells was assessed by an MTT assay, as previously described (15). Briefly, cells were seeded onto 96-well culture plates at a density of 5,000 cells/well. After $24 \mathrm{~h}$, cells were treated with berberine at a concentration of $25,50,100$ or $200 \mu \mathrm{M}$, or with DMSO alone (control group) for 24, 48 and $72 \mathrm{~h}$. After the designated period, $20 \mu \mathrm{l}$ MTT $(5 \mathrm{mg} / \mathrm{ml})$ was added to each well and the plates were cultured at $37^{\circ} \mathrm{C}$ for additional $4 \mathrm{~h}$. Formazan crystals that formed in the wells were dissolved in $150 \mu \mathrm{l}$ DMSO, and the absorbance value of each well was measured at $490 \mathrm{~nm}$ using a Spectra Max M2 spectrophotometer (Molecular Devices, Sunnyvale, CA, USA). All experiments were performed in triplicate.

Detection of apoptosis. To investigate whether the inhibition of cell proliferation by berberine was mediated by apoptosis, we used Hoechst 33258 staining to detect apoptosis. After treatment with berberine at a concentration of 0 (DAMO alone; control group) or $100 \mu \mathrm{M}$, chromatin morphologic changes were observed by fluorescence microscopy after DNA staining with $10 \mu \mathrm{g} / \mathrm{ml}$ Hoechst 33258 (Beijing Solarbio Science \& Technology Co., Ltd., Beijing, China). Reduced nuclear size, chromatin condensation, intense fluorescence, and nuclear fragmentation were considered to indicate apoptotic cells. Then, we further detected the apoptosis rate using Annexin V-PE/7-AAD double staining assay by flow cytometry. The cells were treated with berberine at the concentration of 0 (DMSO alone; control group), or $100 \mu \mathrm{M}$; or the cells were treated with $100 \mu \mathrm{M}$ berberine in the absence or presence of 3-MA, respectively, for $48 \mathrm{~h}$ and then harvested and resuspended in Annexin $\mathrm{V}$ binding buffer. The suspension was incubated with $5 \mu \mathrm{l}$ of Annexin V-PE and $5 \mu \mathrm{l}$ of 7-AAD for $15 \mathrm{~min}$ at room temperature in the dark followed by addition of $400 \mu \mathrm{l}$ of binding buffer. Then the samples were detected immediately by flow cytometry (FACSAria III;
BD Biosciences, San Diego, CA, USA) analysis. All experiments were carried out in triplicate.

Immunofluorescence analysis of LC3 distribution. NCI-H2452 cells were treated with berberine at a concentration of 0 (DMSO alone; control group) or $100 \mu \mathrm{M}$ for $48 \mathrm{~h}$. Then, the treated cells were fixed in $4 \%$ paraformaldehyde for $15 \mathrm{~min}$. After blocking with $5 \%$ normal goat serum and $0.3 \%$ Triton $\mathrm{X}-100$ in phosphate-buffered saline (PBS), the cells were incubated with an anti-LC3B antibody (1:50; cat. no. CST-2775s; Cell Signaling Technology, Inc., Danvers, MA, USA) at $4^{\circ} \mathrm{C}$ overnight, followed by incubation with a TRITC-conjugated goat anti-rabbit IgG secondary antibody (1:200; cat. no. BA1090; Wuhan Boster Biological Technology, Ltd., Wuhan, China) for $1 \mathrm{~h}$ at room temperature. Nuclei were stained with DAPI (Beyotime Institute of Biotechnology, Shanghai, China) for $10 \mathrm{~min}$. Coverslips were mounted in antifade mounting medium and images were obtained using a Leica $^{\mathrm{TM}}$ fluorescence microscope equipped with a Leica ${ }^{\mathrm{TM}}$ camera (Leica Camera AG, Wetzlar, Germany).

Western blot analysis. Expression levels of LC3, p62 and proteins associated with the mitochondrial pathway were measured in NCI-H2452 cells via immunoblot analysis. The cells were treated as described in the Figure legends. Whole cell extracts were prepared in ice-cold lysis buffer. To detect the expression of cytochrome $c$, cytosolic and mitochondrial fractions were prepared using a Mitochondria Extraction kit (Nanjing KeyGen Biotech., Co., Ltd., Nanjing, China). After removal of the insoluble fraction by centrifuging at $20,000 \mathrm{x} \mathrm{g}$ for $30 \mathrm{~min}$ at $4^{\circ} \mathrm{C}$, the protein content of the supernatant was determined via the bicinchoninic acid (BCA) method with a commercial protein assay reagent (CoWin Bioscience Co., Ltd., Beijing, China). Protein samples $(30 \mu \mathrm{g})$ were subjected to precast $8-12 \%$ SDS-PAGE. After electrophoresis, the proteins were transferred to PVDF membranes (EMD Millipore, Billerica, MA, USA). The membranes were then incubated with primary antibodies against LC3B (1:750), p62 (1:1,000), cytochrome $c(1: 1,000)$, caspase-9 $(1: 1,000)$, caspase-3 $(1: 1,000)$, PARP [poly(ADP-ribose) polymerase] $(1: 1,000)$, and Cox 4 $(1: 5,000)$ in $5 \%$ BSA in TBS-T (Cell Signaling Technology, Inc.) and $\beta$-actin (1:1,000; Zhongshan Bio., Beijing, China) overnight at $4^{\circ} \mathrm{C}$. After incubation with horseradish peroxidase (HRP)-conjugated secondary antibodies (Zhongshan Bio.) at a dilution of 1:5,000 in TBS-T for $1 \mathrm{~h}$ at room temperature, the immunoreactive bands were detected using electrochemiluminescence (ECL; EMD Millipore) and quantified using Multi Gauge V3.2 analysis software (Fuji Film, Tokyo Japan).

Trypan blue exclusion assay. Cell death was evaluated using a trypan blue exclusion assay as described previously (13). Briefly, NCI-H2452 cells were cultured for $48 \mathrm{~h}$ with various concentrations of berberine, or with $200 \mu \mathrm{M}$ berberine in the absence or presence of pan-caspase inhibitor z-VAD-fmk $(20 \mu \mathrm{M})$, autophagy inhibitor 3-MA (5 mM), or autophagy inducer rapamycin $(100 \mathrm{nM})$. Then, both adherent and non-adherent cells were collected, washed three times with PBS, and resuspended in PBS at a concentration of $1 \times 10^{6}$ cells $/ \mathrm{ml}$. After mixing with $0.4 \%$ trypan blue (ratio of cells:trypan blue, 9:1), the cells were counted using a hemocytometer. The number of 


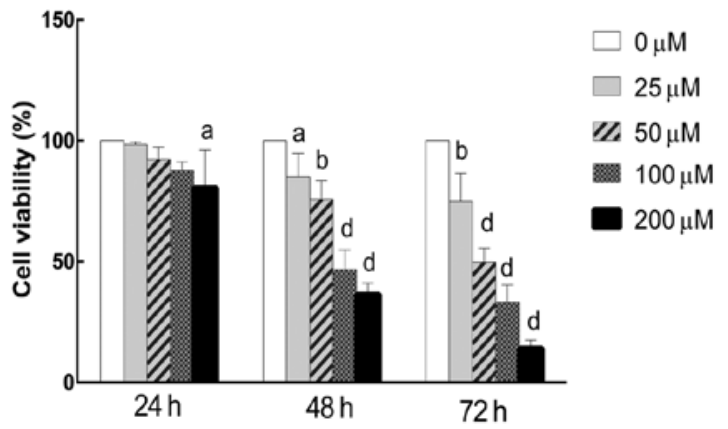

Figure 1. MTT assays show the inhibitory effect of berberine on NCI-H2452 cell growth. NCI-H2452 cells were treated with berberine at a concentration of $0,25,50,100$ or $200 \mu \mathrm{M}$ for 24,48 and $72 \mathrm{~h}$. Berberine inhibited the proliferation of NCI-H2452 cells in a dose- and time-dependent manner. ${ }^{a} \mathrm{P}<0.05$ vs. control, ${ }^{b} \mathrm{P}<0.01$ vs. control, ${ }^{\mathrm{d}} \mathrm{P}<0.001$ vs. control.

dead cells with disrupted membranes (blue cells) was counted. The cell death rate was calculated as the mean percentage of blue cells/total cells. All experiments were conducted in triplicate.

Statistical analysis. All data in this study are expressed as the means $\pm \mathrm{SD}$. A one-way analysis of variance (ANOVA) or t-test was performed to assess differences between groups under different conditions. All statistical analyses were performed using SPSS 19.0 software (IBM Corp., Armonk, NY, USA). $\mathrm{P}<0.05$ was considered statistically significant.

\section{Results}

Berberine inhibits the proliferation of NCI-H2452 cells in a dose- and time-dependent manner. The antiproliferation effect of berberine against MPM NCI-H2452 cells was evaluated by an MTT assay. The results showed that berberine inhibited the growth of cells in a dose- and time-dependent manner. The proliferation rate of berberine-treated NCI-H2452 cells was $87.58 \pm 3.72,46.68 \pm 8.08$ and $33.24 \pm 7.17 \%$, respectively, following treatment with berberine at a concentration of $100 \mathrm{mM}$ for 24, 48 and $72 \mathrm{~h}$ (Fig. 1). Based on the MTT assay results, the $100 \mu \mathrm{M}$ berberine concentration for $48 \mathrm{~h}$ was used in subsequent experiments.

Berberine induces the apoptosis of NCI-H2452 cells. Previous studies have reported that berberine can induce apoptosis in some cancer cells $(9,10)$; therefore, we investigated whether it could induce apoptosis in NCI-H2452 cells using Hoechst 33258 staining. As shown in Fig. 2A, the treatment of NCI-H2452 cells with berberine at $100 \mu \mathrm{M}$ for $48 \mathrm{~h}$ resulted in apoptosis, which was characterized by unique morphological nuclear changes such as nuclear condensation and fragmentation.
A

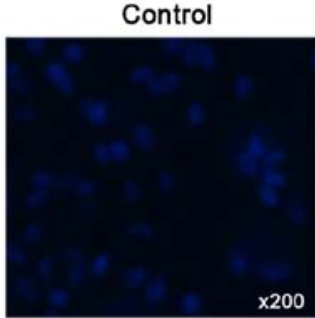

B

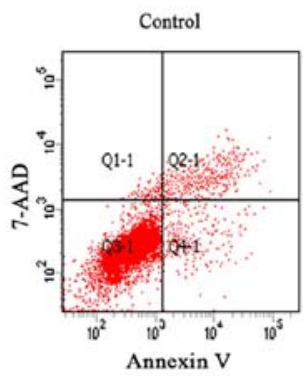

Berberine

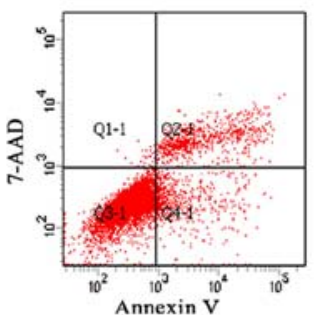

Berberine

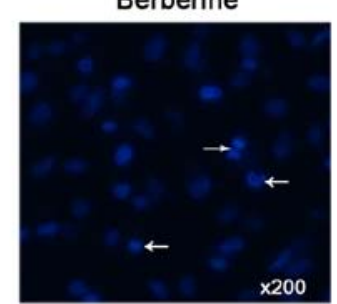

C

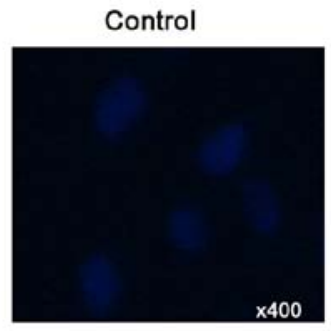

D

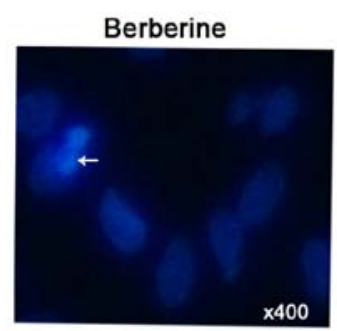

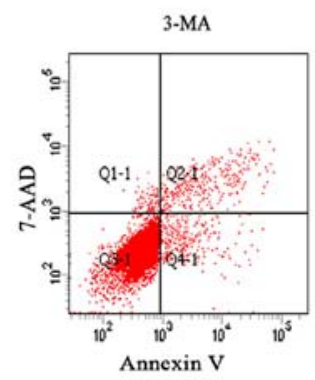

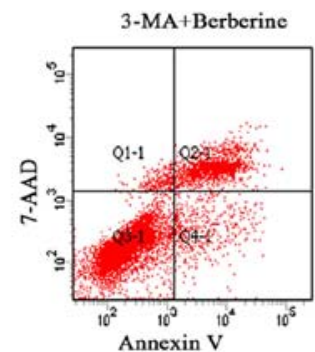

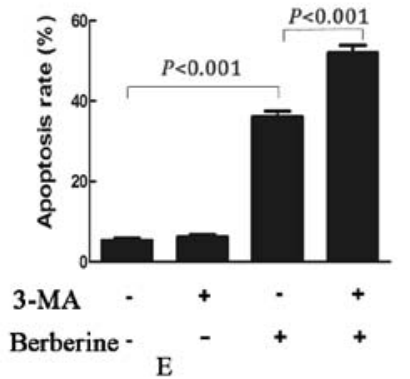

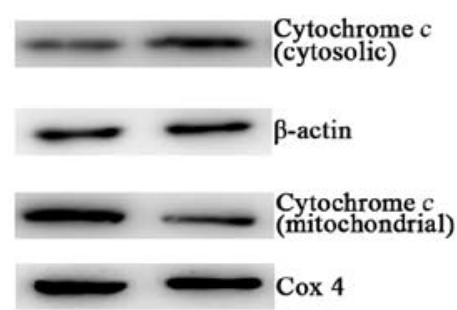

Figure 2. Effects of berberine on NCI-H2452 cell apoptosis. Cells were treated with berberine at a concentration of $0 \mu \mathrm{M}$ (control group; DMSO only) or $100 \mu \mathrm{M}$ (berberine group); or the cells were treated with $100 \mu \mathrm{M}$ berberine in the absence or presence of 3-MA, respectively, for 48 h. (A) Apoptotic cancer cells stained by Hoechst 33258 are indicated with an arrow (A, x200 and x400 magnification). (B and C) The apoptosis rate was detected by Annexin V-PE/7-AAD analysis; the apoptosis rate was calculated as the percentage of early apoptosis+late apoptosis. (D and E) Western blot analysis of cytochrome $c$, caspase-9, caspase- 3 and PARP expression in berberine-treated and control cells. 


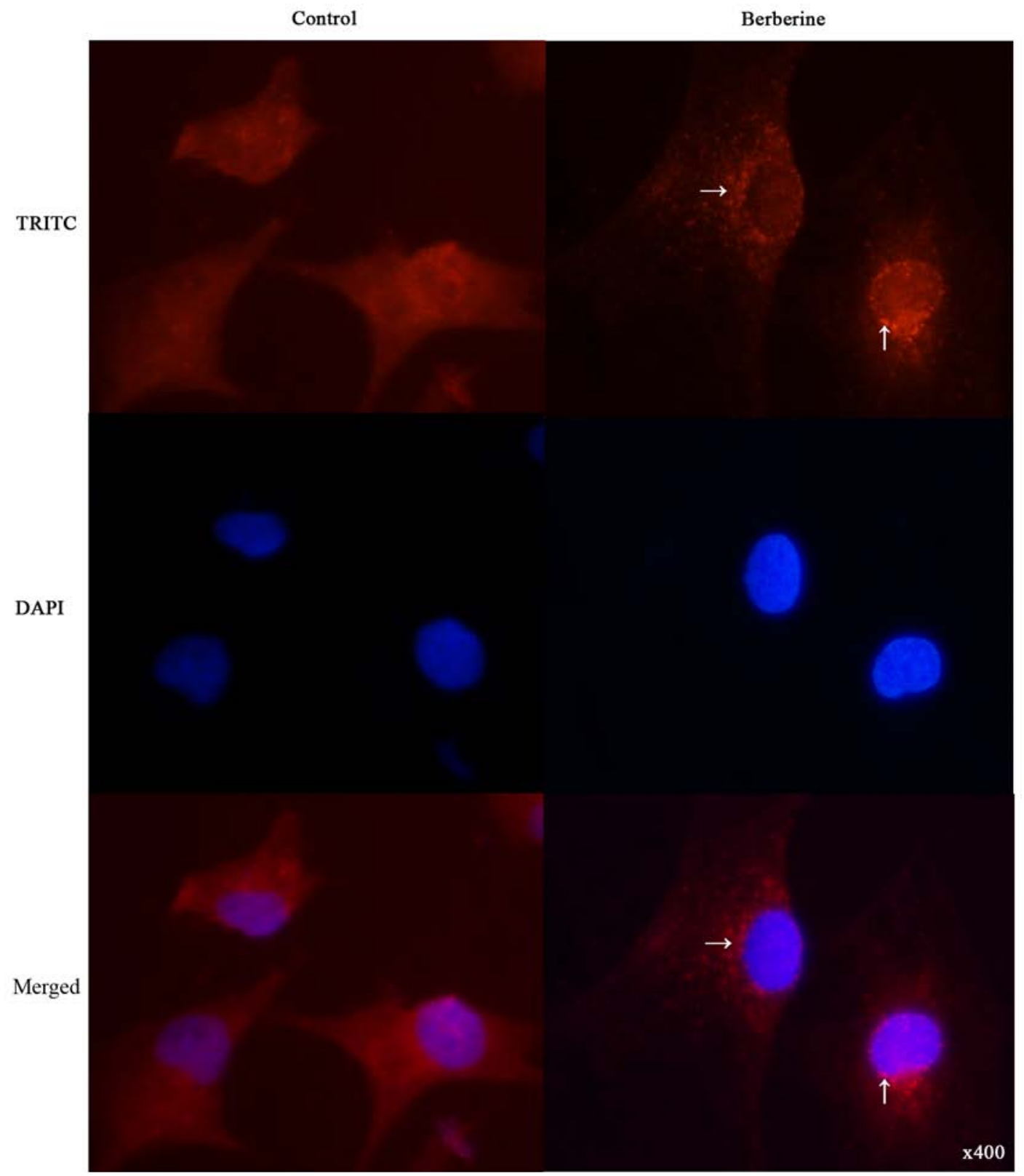

Figure 3. Immunofluorescence images showing the redistribution of the autophagy marker LC3 in NCI-H2452 cells treated with berberine at the concentration of $0 \mu \mathrm{M}$ (control group; DMSO only) or $100 \mu \mathrm{M}$ (berberine group) for $48 \mathrm{~h}$ were taken under a Leica ${ }^{\mathrm{TM}}$ fluorescence microscope equipped with a Leica ${ }^{\mathrm{TM}}$ camera (magnification, $\mathrm{x} 400$ ).

We further examined the apoptosis rate by Annexin V-PE/ 7-AAD double staining assay by flow cytometry. The results showed that after treatment with berberine at the concentration of $100 \mu \mathrm{M}$ for $48 \mathrm{~h}$, the apoptosis rate of NCI-H2452 cells increased to $32.23 \pm 1.06 \%$, while the apoptosis rate was $3.55 \pm 0.86 \%$ in untreated cells $(\mathrm{P}<0.001$; Fig. $2 \mathrm{~B}$ and $\mathrm{C})$.

Berberine-induced apoptosis is mediated by a mitochondrial pathway involving caspase-9. Many antitumor agents induce cancer cells apoptosis through mitochondrial apoptotic pathways $(16,17)$; therefore, we further investigated the expression of key proteins in the mitochondrial pathway. As shown in Fig. 2D and E, treatment with berberine resulted in the release of cytochrome $c$ into the cytoplasm, as well as a significant increase in the active forms of caspase- 9 and caspase- 3 , and the proteolytic cleavage of poly(ADP-ribose) polymerase (PARP).
Berberine induces autophagy in NCI-H2452 cells. To determine whether berberine induces autophagy in NCI-H2452 cells, we examined the intracellular distribution of LC3, an autophagy marker, upon berberine treatment under immunofluorescence. As shown in Fig. 3, the distribution of LC3 fluorescence changed from diffuse cytosolic in untreated cells to punctate upon berberine treatment.

We further analyzed the expression of LC3-II using western blotting (Fig. 4A and B). The results showed an increased conversion of LC3-I to the autophagic LC3-II isoform in NCI-H2452 cells treated with berberine, in a dose- and time-dependent manner. The increased conversion was attenuated by pre-treatment with the autophagic inhibitor 3-MA, and enhanced by the autophagic inducer rapamycin (Fig. 5A and B). In addition, treatment with berberine decreased the expression of p62 in a dose- and time-dependent manner (Fig. 4A and B). 
A

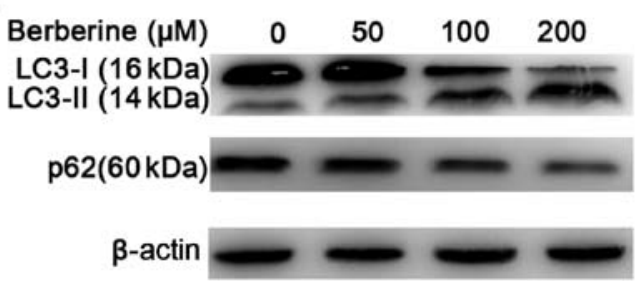

B

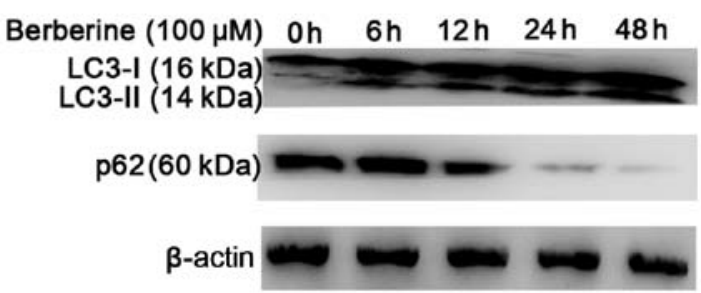

Figure 4. (A) Western blot analysis of LC3-II and p62 expression in NCI-H2452 cells treated with berberine at a concentration of $0,50,100$ or $200 \mu \mathrm{M}$ for $48 \mathrm{~h}$. (B) Western blot analysis of LC3-II and p62 expression in NCI-H2452 cells treated with berberine at $100 \mu \mathrm{M}$ for various time intervals.

A

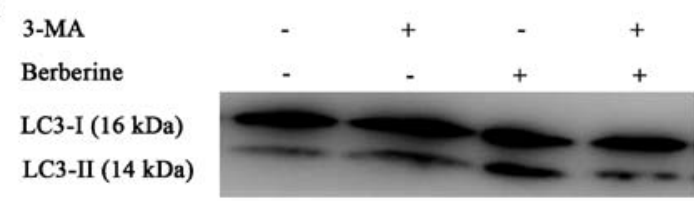

$\beta-\operatorname{actin}(43 \mathrm{kDa})$

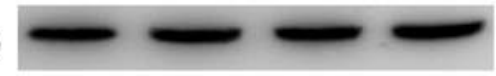

B

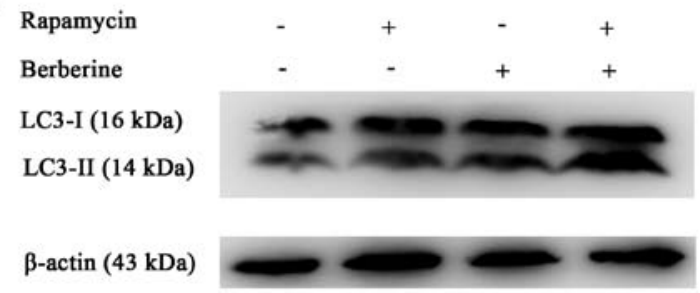

Figure 5. Western blot analysis of LC3-II expression in NCI-H2452 cells treated with berberine at $100 \mu \mathrm{M}$ in the absence or presence of (A) 3-MA or (B) rapamycin.

These results suggest that autophagy is induced by berberine in NCI-H2452 cells.

Berberine induces cell death in a dose-dependent manner. Cell death in response to berberine was quantified by a trypan blue exclusion assay, and the cells with disrupted membranes (blue cells) were counted as dead cells. NCI-H2452 cells were treated with berberine at concentrations of $0,50,100$ and $200 \mu \mathrm{M}$ for 48 h. As shown in Fig. 6, berberine killed MPM cells in a dose-dependent manner. After treatment with berberine at the concentration of $200 \mu \mathrm{M}$ for $48 \mathrm{~h}$, the death rate of NCI-H2452 cells increased to $34.66 \pm 5.32 \%(\mathrm{P}<0.001)$.

Our western blot results showed that autophagy was induced in NCI-H2452 cells by treatment with berberine in a dose-dependent manner. Based on the western blotting results, autophagy was induced at a concentration of $200 \mu \mathrm{M}$. As cell death was clearly induced with a concentration of $200 \mu \mathrm{M}$ berberine in the trypan blue exclusion assay, this concentration was used in subsequent experiments.

Protective autophagy during berberine treatment. The results showed that MPM cells treated with berberine underwent autophagy in a dose- and time-dependent manner, which was positively correlated with the cytotoxic activity of berberine; therefore, we further assessed whether berberine-induced NCI-H2452 cell death was mediated by autophagy. In addition,

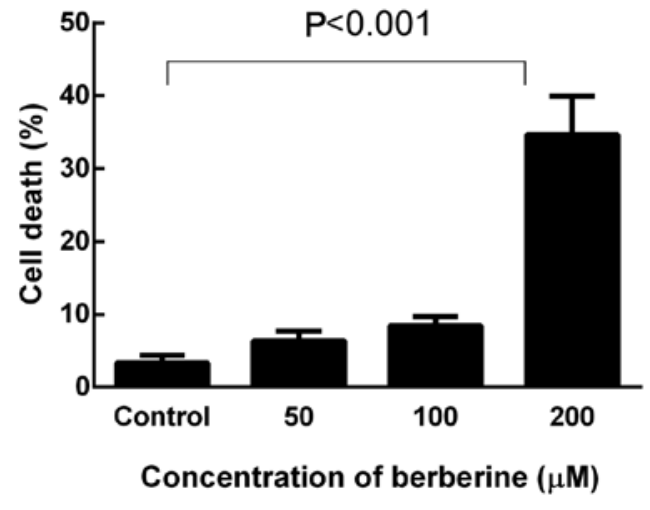

Figure 6. NCI-H2452 cell death in response to treatment with berberine at various concentrations for $48 \mathrm{~h}$ was assessed using a trypan blue exclusion assay. Results are expressed as the means $\pm \mathrm{SD}, \mathrm{n}=3$.

both apoptosis and autophagy were induced by berberine treatment in this study. Therefore, it was necessary to further investigate the interactions between berberine-induced apoptosis and autophagy. We first explored whether the inhibition of autophagy by 3-MA or the induction of autophagy by rapamycin affected the rate of cell death induced by berberine (Fig. 7A and B). The results showed that the death rate of NCI-H2452 cells treated with 3-MA plus berberine increased significantly compared with the cells treated with berberine alone, while pre-treatment with rapamycin decreased the death rate of berberine-treated cells.

The apoptosis rate of NCI-H2452 cells was assessed using Annexin V-PE/7-AAD double staining assay by flow cytometry. Compared with the cells treated with berberine alone, 3-MA-treated cells underwent extensive apoptosis after berberine treatment (Fig. 2B and C). However, the expression level of LC3-II in NCI-H2452 cells treated with berberine in the presence of $\mathrm{z}-\mathrm{VAD}$-fmk was similar to that in the cells treated with berberine alone (Fig. 7C).

Subsequently, we further investigated if berberine-induced NCI-H2452 cell death occurs via apoptosis. Cells were pretreated with z-VAD-fmk, a pan-caspase inhibitor, which only inhibits apoptosis-mediated cell death, for $1 \mathrm{~h}$, followed by berberine for $48 \mathrm{~h}$. As shown in Fig. 7D, berberine-induced NCI-H2452 cell death was attenuated by z-VAD-fmk.

Collectively, the results show that apoptosis is the main route by which berberine induces NCI-H2452 cell death. Contrary to previous studies, our results supported a cytoprotective autophagic activity induced by berberine, preventing cells from undergoing apoptosis; the inhibition of autophagy 
A

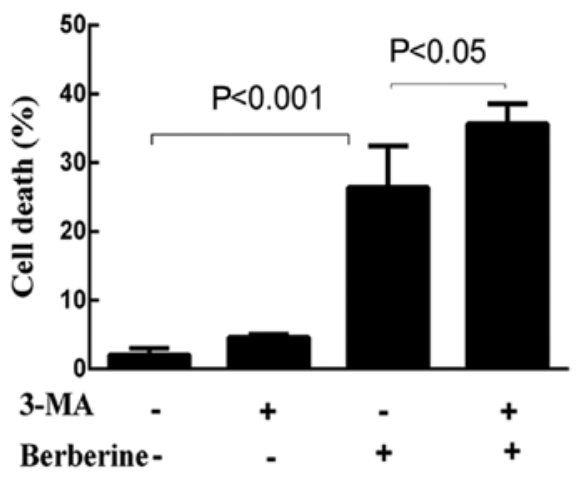

C

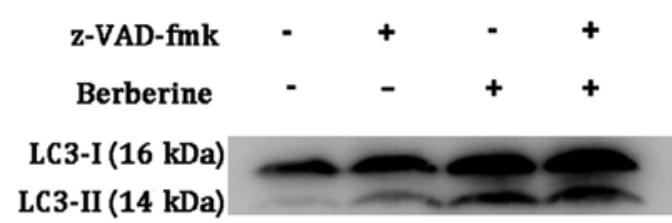

$\beta$-actin (43 kDa)
B
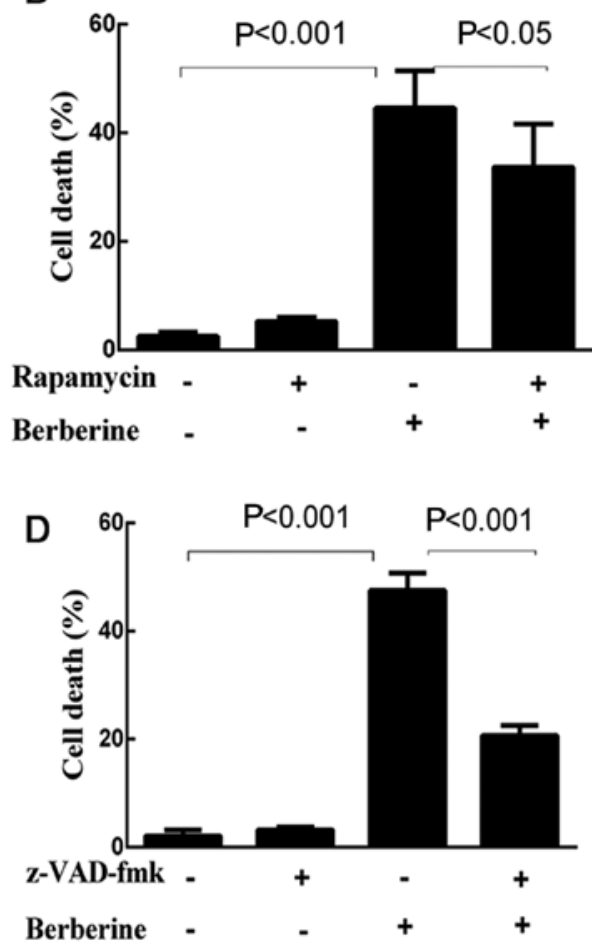

Figure 7. Effects of apoptosis and autophagy in berberine-induced cell death. (A and B) NCI-H2452 cells were treated for $48 \mathrm{~h}$ with berberine (200 $\mu \mathrm{M})$ in the presence or absence of 3-MA $(5 \mathrm{mM})$ or rapamycin $(100 \mathrm{nM})$. (C) Western blot analysis of LC3-II expression in NCI-H2452 cells treated with berberine at $100 \mu \mathrm{M}$ in the absence or presence of z-VAD-fmk $(20 \mu \mathrm{M})$ for $48 \mathrm{~h}$. (D) NCI-H2452 cells were treated for $48 \mathrm{~h}$ with berberine (200 $\mu \mathrm{M})$ in the presence or absence of z-VAD-fmk $(20 \mu \mathrm{M})$. Results are expressed as the means $\pm \mathrm{SD}, \mathrm{n}=3$.

may enhance the antitumor potential of berberine in MPM NCI-H2452 cells.

\section{Discussion}

It has been shown that berberine has potent antitumor activity in many types of tumor cells both in vitro and in vivo, while having no significant inhibitory effect on normal cells (18). In the present study, we investigated the underlying molecular mechanisms of berberine-induced MPM cell death. Our results suggest that berberine possesses cytotoxic effects against MPM in vitro. Berberine clearly suppressed proliferation and induced apoptosis in NCI-H2452 cells.

Malignant cells are frequently resistant to apoptotic stimuli, and a blockade of apoptosis may promote cancer progression. The intrinsic mitochondrial pathway is key to apoptosis. Cytochrome $c /$ caspase- 9 activation is an initial event in the mitochondrial pathway. Cytochrome $c$ is an apoprotein that is nearly undetectable in the cytosol of normal cells (19). However, release of cytochrome $c$ from mitochondria into the cytosol can be induced when the mitochondrial pathway is activated. Apoptotic protease-activating factor-1 (Apaf1) and cytochrome $c$ are both involved in the activation of caspase-9 (20). In turn, activated caspase- 9 induces procaspase- 3 to form activated caspase-3, which results in cleavage and inactivation of the DNA repair enzyme poly(ADP-ribose polymerase) (21). In the present study, we found that cytosolic cytochrome $c$ was upregulated by treatment with berberine. Activated caspase-9, activated caspase- 3 and cleaved PARP were observed in berberine-treated cells. These results suggest that berberine-induced apoptosis may occur via cytochrome $c /$ caspase-9/caspase-3 signaling in MPM NCI-H2452 cells.

Recently, studies report that berberine can exert antitumor effects not only through apoptosis, but also through autophagy in hepatic and colon cancer cell lines. Autophagy is a highly evolutionarily conserved process common in eukaryotes. It is a lysosomal degradation process in which long-lived proteins and damaged or aged cellular organelles are encapsulated within autophagosomes and degraded by lysosomal hydrolases to maintain cell homeostasis under physiological conditions. While under pathological conditions, autophagy is generally recognized to serve protective effects. However, excessive autophagy may lead to autophagic cell death, also called type II programmed cell death. Recently, increasing evidence has shown that autophagy has an important role in tumorigenesis, development and tumor suppression. Autophagy has been observed in response to antitumor drugs such as arsenic trioxide, matrine, bufarin and resveratrol, suggesting that autophagy can be used as a tumor treatment strategy.

However, the role of autophagy in tumor treatment is still controversial. Some studies have shown that autophagy induced by antitumor agents has pro-survival effects and decreases drug efficacy. Autophagy is recognized as one of the mechanisms involved in the drug resistance of cancer cells. Matrine, resveratrol and quercetin (22) induce protective autophagy in many cancer cell types, and acquired cisplatin-resistance in human lung adenocarcinoma cells is associated with enhanced autophagy (23).

Various studies have demonstrated that certain antitumor agents induce cell death through autophagy; thus, suppression 
of autophagy can decrease drug cytotoxicity. In addition, previous studies report that autophagy induced by the same agent in different cancer cells led to different effects. Therefore, when we evaluate the activity of one antitumor agent, we need to investigate whether autophagy is activated during treatment and further explore the role of autophagy in drug-induced cytotoxicity.

In the present study, we found that berberine is a strong inducer of apoptosis and autophagy. The expression of LC3-II, an autophagic marker, increased in a dose- and time-dependent manner after treatment with berberine. The cell death rate also increased in a dose-dependent manner. Based on these results, we proposed two hypotheses: Berberine-induced autophagy may be a mechanism of antitumor activity, leading to autophagic cell death; or it may be a mechanism by which protective effects are conferred onto MPM cells during berberine treatment. Therefore, we used inhibitors of apoptosis or autophagy, and an inducer of autophagy, to evaluate the significance of autophagy in berberine-induced cell death. Our data indicated that the presence of autophagy inhibitor 3-MA augmented berberine-induced NCI-H2452 cell death, while the autophagy inducer rapamycin and apoptosis inhibitor z-VAD-fmk attenuated berberine-induced NCI-H2452 cell death. An Annexin V-PE/7-AAD double staining assay indicated that the inhibition of autophagy by 3-MA enhanced berberine-induced apoptosis, suggesting that berberine-induced autophagy protects cells from apoptosis and, thus, might be a target for enhancing its antitumor activity. In addition, treatment with berberine plus 3-MA significantly enhanced the antiproliferation effects against NCI-H2452 cells, relative to berberine alone.

The aforementioned results revealed that apoptosis is the main form of death induced by berberine in MPM NCI-H2452 cells. Additionally, autophagy might have a protective response through the degradation and recycling of damaged cellular organelles and proteins caused by berberine treatment, which protects MPM cells from berberine-induced apoptosis.

Our results were inconsistent with studies of berberine in hepatocarcinoma cell lines conducted by Hou et al (13) and Wang et al (24). Their results showed that berberine induced cell death through both apoptosis and autophagy, and that the inhibition of autophagy decreased cell death. This may be because the effects were studied in a different cell type. Therefore, the results need to be validated in more cell lines and in vivo studies in the future.

However, our study had limitations. According to previous literature (25), increases in the level of LC3-II, can reflect the induction of autophagy and/or inhibition of autophagosome or amphisome clearance. Autophagic flux reflects the entire process of autophagy. Thus, at present, the actual mechanistic relationship between LC3-II formation and the rest of the autophagic process is not known; indeed, it may be possible to execute 'self-eating' in the absence of LC3-II. It is more appropriate to detect autophagy based on autophagic flux. Lysosomal degradation is an important part of the autophagy pathway. This has been included in our other study that is currently being conducted, and thus was not included in the present study.

In conclusion, the present study demonstrated that berberine is a potent antitumor agent for treating MPM. It can induce apoptosis, possibly through a caspase-9-dependent intrinsic mitochondrial pathway. Berberine can also induce autophagy in NCI-H2452 cells, although apoptosis is the major form of berberine-induced NCI-H2452 cell death. Berberine-induced autophagy may be an adaptive response to antitumor agents and have a protective role in MPM cells. Inhibition of autophagy enhanced berberine-induced apoptosis. Therefore, the inhibition of autophagy may be an effective treatment strategy for the management of MPM.

\section{Acknowledgements}

Not applicable.

\section{Funding}

The present study was funded by the Natural Science Foundation of Shandong Province of China (grant no. ZR2016HL27).

\section{Availability of data and materials}

The analyzed data sets generated during the study are available from the corresponding author on reasonable request.

\section{Authors' contributions}

DL and QL designed the study. ZY conducted the experiments and analyzed the data. YW and BL assisted with the western blot analysis. CZ and FY assisted with the immunofluorescence experiments and the analysis. YK assisted with collecting and analyzing the data. All authors read and approved the final manuscript and agree to be accountable for all aspects of the research in ensuring that the accuracy or integrity of any part of the work are appropriately investigated and resolved.

\section{Ethics approval and consent to participate}

Not applicable.

\section{Patient consent for publication}

Not applicable.

\section{Competing interests}

The authors declare that they have no competing interests.

\section{References}

1. Wagner JC, Sleggs CA and Marchand P: Diffuse pleural mesothelioma and asbestos exposure in the North Western Cape Province. Br J Ind Med 17: 260-271, 1960.

2. Hodgson JT, McElvenny DM, Darnton AJ, Price MJ and Peto J: The expected burden of mesothelioma mortality in Great Britain from 2002 to 2050 . Br J Cancer 92: 587-593, 2005.

3. Liu JC, Chan P, Chen YJ, Tomlinson B, Hong SH and Cheng JT: The antihypertensive effect of the berberine derivative 6-protoberberine in spontaneously hypertensive rats. Pharmacology 59: 283-289, 1999.

4. Moghaddam HK, Baluchnejadmojarad T, Roghani M, Khaksari M, Norouzi P, Ahooie M and Mahboobi F: Berberine ameliorate oxidative stress and astrogliosis in the hippocampus of STZ-induced diabetic rats. Mol Neurobiol 49: 820-826, 2014.

5. Zhou ZW, Zheng HC, Zhao LF, Li W, Hou JW, Yu Y, Miao PZ and Zhu JM: Effect of berberine on acetylcholine-induced atrial fibrillation in rabbit. Am J Transl Res 7: 1450-1457, 2015. 
6. Zhang XJ, Deng YX, Shi QZ, He MY, Chen B and Qiu XM Hypolipidemic effect of the Chinese polyherbal Huanglian Jiedu decoction in type 2 diabetic rats and its possible mechanism. Phytomedicine 21: 615-623, 2014

7. Kim S, Lee J, You D, Jeong Y, Jeon M, Yu J, Kim SW, Nam SJ and Lee JE: Berberine suppresses cell motility through downregulation of TGF- $\beta 1$ in triple negative breast cancer cells. Cell Physiol Biochem 45: 795-807, 2018.

8. Li F, Dong X, Lin P and Jiang J: Regulation of Akt/FoxO3a/Skp2 axis is critically involved in berberine-induced cell cycle arrest in hepatocellular carcinoma cells. Int J Mol Sci 19: pii: E327, 2018.

9. Okubo S, Uto T, Goto A, Tanaka H, Nishioku T, Yamada K and Shoyama Y: Berberine induces apoptotic cell death via activation of caspase-3 and -8 in HL-60 human leukemia cells: Nuclear localization and structure-activity relationships. Am J Chin Med 45: 1497-1511, 2017.

10. Zhao Y, Jing Z, Lv J, Zhang Z, Lin J, Cao X, Zhao Z, Liu P and Mao W: Berberine activates caspase-9/cytochrome $c$-mediated apoptosis to suppress triple-negative breast cancer cells in vitro and in vivo. Biomed Pharmacother 95: 18-24, 2017.

11. Jiang SX, Qi B, Yao WJ, Gu CW, Wei XF, Zhao Y, Liu YZ and Zhao BS: Berberine displays antitumor activity in esophageal cancer cells in vitro. World J Gastroenterol 23: 2511-2518, 2017.

12. Wang X, Wang N, Li H, Liu M, Cao F, Yu X, Zhang J, Tan Y, Xiang L and Feng Y: Up-regulation of PAI-1 and down-regulation of UPA are involved in suppression of invasiveness and motility of hepatocellular carcinoma cells by a natural compound berberine. Int J Mol Sci 17: 577, 2016.

13. Hou Q, Tang X, Liu H, Tang J, Yang Y, Jing X, Xiao Q, Wang W, Gou $X$ and Wang Z: Berberine induces cell death in human hepatoma cells in vitro by downregulating CD147. Cancer Sci 102: 1287-1292, 2011.

14. La X, Zhang L, Li Z, Yang P and Wang Y: Berberine-induced autophagic cell death by elevating GRP78 levels in cancer cells. Oncotarget 8: 20909-20924, 2017.

15. Kang Y, Ding M, Tian G, Guo H, Wan Y, Yao Z, Li B and Lin D: Overexpression of Numb suppresses tumor cell growth and enhances sensitivity to cisplatin in epithelioid malignant pleural mesothelioma. Oncol Rep 30: 313-319, 2013.
16. Patil JB, Kim J and Jayaprakasha GK: Berberine induces apoptosis in breast cancer cells (MCF-7) through mitochondrial-dependent pathway. Eur J Pharmacol 645: 70-78, 2010.

17. Yang $X$ and Huang N: Berberine induces selective apoptosis through the AMPK-mediated mitochondrial/caspase pathway in hepatocellular carcinoma. Mol Med Rep 8: 505-510, 2013.

18. Mantena SK, Sharma SD and Katiyar SK: Berberine, a natural product, induces $\mathrm{G}_{1}$-phase cell cycle arrest and caspase-3dependent apoptosis in human prostate carcinoma cells. Mol Cancer Ther 5: 296-308, 2006.

19. Renz A, Berdel WE, Kreuter M, Belka C, Schulze-Osthoff K and Los M: Rapid extracellular release of cytochrome $c$ is specific for apoptosis and marks cell death in vivo. Blood 98: 1542-1548, 2001.

20. Zou H, Li Y, Liu X and Wang X: An APAF-1.cytochrome $c$ multimeric complex is a functional apoptosome that activates procaspase-9. J Biol Chem 274: 11549-11556, 1999.

21. Wolf BB and Green DR: Suicidal tendencies: Apoptotic cell death by caspase family proteinases. J Biol Chem 274: 20049-20052, 1999.

22. Kim H, Moon JY, Ahn KS and Cho SK: Quercetin induces mitochondrial mediated apoptosis and protective autophagy in human glioblastoma U373MG cells. Oxid Med Cell Longev 2013: 596496, 2013.

23. Ren JH, He WS, Nong L, Zhu QY, Hu K, Zhang RG, Huang LL, Zhu F and Wu G: Acquired cisplatin resistance in human lung adenocarcinoma cells is associated with enhanced autophagy. Cancer Biother Radiopharm 25: 75-80, 2010.

24. Wang N, Feng Y, Zhu M, Tsang CM, Man K, Tong Y and Tsao SW: Berberine induces autophagic cell death and mitochondrial apoptosis in liver cancer cells: The cellular mechanism. J Cell Biochem 111: 1426-1436, 2010.

25. Klionsky DJ, Abdelmohsen K, Abe A, Abedin MJ, Abeliovich H, Acevedo Arozena A, Adachi H, Adams CM, Adams PD, Adeli K, et al: Guidelines for the use and interpretation of assays for monitoring autophagy (3rd edition). Autophagy 12: 1-222, 2016. 